\title{
Characteristics of cerebrospinal fluid (CSF) among children with ventriculoperitoneal shunt infections
}

\author{
Hernán Rowensztein, M.D, ${ }^{a}$ Lisandro Manfrin, M.D., ${ }^{a}$ Marcela Paglia, M.D., ${ }^{a}$ Tai Luong Cong, M.D., ${ }^{a}$ \\ Silvina Ruvinsky, M.D., ${ }^{b}$ and Adriana Scrigni, M.D. ${ }^{a}$
}

\begin{abstract}
A common (5\%) complication of cerebrospinal fluid (CSF) shunt systems is infection. CSF cytochemical findings have been used to choose the initial empirical antimicrobial treatment. The objective was to evaluate the results of cytochemical and microbiological analyses of CSF samples obtained from children with pyogenic ventriculitis associated to ventriculoperitoneal shunt systems. This was a retrospective, descriptive and analytical study. Thirty-two cases of pyogenic ventriculitis were included; Gram-positive bacteria were predominant in 26 cases $(81.25 \%)$; the rest corresponded to Gram-negative bacteria. There were no statistically significant differences for the studied outcome variables (CSF cellularity, glucose and protein levels).

Conclusion: Gram-positive bacteria were widely predominant as a cause of pyogenic ventriculitis associated to the surgical procedure. CSF characteristics show a high heterogeneity and were not associated to the type of microorganism in this studied sample.

Key words: cerebral ventriculitis, ventriculoperitoneal shunt, cerebrospinal fluid, hydrocephalus.
\end{abstract}

http:/ /dx.doi.org/10.5546/aap.2015.eng.244

\section{INTRODUCTION}

Excessive production of cerebrospinal fluid (CSF) and / or obstructed CSF flow lead to hydrocephalus ${ }^{1}$ and are the most common causes for CSF shunt placement. CSF shunts are now commonly used in pediatrics given the survival of preterm infants who suffer from intraventricular hemorrhage. Shunts are also used for other types of hydrocephalus, such as hydrocephalus secondary to central nervous system (CNS) tumors, congenital hydrocephalus, etc.

a. Department of Clinical Pediatrics in ModerateIntermediate Care.

b. Department of Epidemiology and Infectology. Hospital de Pediatría"Prof. Dr. Juan P. Garrahan", Buenos Aires, Argentina.

E-mail Address:

Hernán Rowensztein, M.D.: hrowens@intramed.net

Funding: None.

Conflict of interest: None.

Received: 7-7-2014

Accepted: 12-4-2014
The most feared and common complication is shunt infection associated to the surgical procedure for shunt implantation, which ranges between $0 \%$ and $20 \%$, depending on the series. ${ }^{2}$ Since a definitive CSF culture usually takes 5 to 7 days, CSF cytochemical findings have been used in clinical practice to choose the initial empirical antimicrobial treatment based on previously

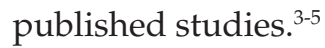

The objective of this study was to evaluate the results of cytochemical and microbiological analyses of CSF samples obtained from children with pyogenic ventriculitis associated to ventriculoperitoneal shunt systems (VPS) and establish the association between cytochemical findings and the type of microorganism.

\section{MATERIAL AND METHODS}

This was a retrospective, descriptive and analytical study conducted at Hospital de Pediatría "Prof. Dr. Juan P. Garrahan," a tertiary care facility located in Buenos Aires, Argentina. Medical records (case history and computer data bases) were reviewed for the period between January 2010 and December 2012. All patients diagnosed with pyogenic ventriculitis associated to the surgical procedure were selected. Ventriculitis was defined according to the criteria established by the Centers for Disease Control and Prevention's National Healthcare Safety Network (CDC/NHSN) 2014, and infections had to be diagnosed in the six months following CSF shunting surgery. ${ }^{6}$ All CSF samples were obtained through valve reservoir puncture; CSF physical, chemical and microbiological findings were collected at the time of the diagnosis of pyogenic ventriculitis.

Data were entered into an Excel spreadsheet and after wards processed using the S.P.S.S. statistical software package v17.0. Histograms were performed to establish the distribution of numerical outcome measures. Based on these histograms, groups were compared using a Mann-Whitney test given their non-parametric distribution. The frequency of occurrence of each microorganism was determined. They were 
then grouped as either Gram-positive or Gramnegative, and CSF cellularity, glucose and protein levels were compared for both groups. A $p$ value lower than 0.05 was considered the cut-off point for statistically significant differences.

\section{RESULTS}

Thirty-two cases (17 males) of documented pyogenic ventriculitis confirmed by microbiological isolation in the studied period were included. These account for approximately $5 \%$ of all VPSs placed in the same period (640 surgical procedures). Patients' age ranged between 3 months and 13 years old.

Their underlying diagnosis, which led to VPS requirement, included CNS tumors $(n=10)$, congenital hydrocephalus $(n=9)$, intraventricular hemorrhage $(n=5)$, Dandy-Walker malformation $(n=2)$, and others, such as meningitis, traumatic brain injury, etc.

In terms of microbiology, all cultures resulted monomicrobial. Gram-positive bacteria were predominant in 26 cases $(81.25 \%)$; the rest corresponded to Gram-negative bacteria. No fungi were observed. The most commonly found bacteria were methicillin-resistant coagulasenegatives staphylococci $(n=17)$, followed by methicillin-sensitive Staphylococcus aureus $(n=5)$ and methicillin-resistant Staphylococcus aureus $(\mathrm{n}=3)$, and then by Pseudomonas aeruginosa $(\mathrm{n}=3)$ and Acinetobacter $(\mathrm{n}=2)$; the rest corresponded to other microorganisms.

Median values and ranges of dispersion for CSF glucose and protein levels and number of WBCs -classified by Gram staining-were compared (shown in Table 1). No statistically significant differences were observed for analyzed outcome measures.

The time elapsed between CSF shunting surgery and diagnosis of pyogenic ventriculitis was also studied. The median time in the Gramnegative group was 27 days (range: 4-139), while it was 28 days (range: 2-151) in the Gram-positive group; no statistically significant differences were observed $(p=0.9)$. The frequency of occurrence of microorganisms was analyzed for the patients in whom infection had been detected in the 15 days following VPS placement, and found that this was the case in 11 Gram-positive infections (42\%) and in half of Gram-negative infections $(\mathrm{n}=3)$; no statistically significant differences were observed (Fisher's two-tailed test $=1$ ).

\section{DISCUSSION}

Infections are the most common complications of CSF shunts. In relation to microorganisms causative of pyogenic ventriculitis, in our hospital it was observed that Gram-positive bacteria are predominant while Gram-negative bacteria have decreased. The trends seen in other hospitals are consistent with our study, as described in the article by Kuo Wei; ${ }^{7}$ however, other hospitals have reported Acinetobacter as the most common etiologic agent (Brazil and Australia). ${ }^{2}$

The study conducted at our site by Grandi L., et al. and published in $1997^{3}$ documented a significant difference in the number of cells that stained Gram-negative, which was higher than the number observed to stain Gram-positive. This was also described for external CSF drainages. ${ }^{5}$ The same was observed in other published studies, ${ }^{8-10}$ although it was not confirmed in our research; however, the values observed by us show a similar trend. Such differences maybe due to the fact that Gram-negative bacteria have remarkably decreased as causative agents of pyogenic ventriculitis, from 30\% in the 1992-1995 period to $19 \%$ at present. In addition, the study mentioned above assumed a normal distribution of outcome measures used parametric tests for comparison; in our case, and given the highly biased distribution of studied outcome measures, non-parametric tests (Mann-Whitney) were used.

Rosanova and Paganini ${ }^{11}$ pointed out that a high CSF cell count correlates with infection, but a normal cell count does not rule it out. The same has been observed in relation to CSF glucose and protein level alterations. It should also be noted that an increased CSF cellularity has been described in the 15 days following VPS placement caused by the surgical trauma itself.

As shown in Table 1, the CSF cellularity range for both Gram-negative and Grampositive bacteria encompasses some cases with a CSF cellularity below 5, which is the cut-off point for normality. This is important because there maybe cases of pyogenic ventriculitis with normal CSF cellularity that would require a strong diagnostic suspicion to initiate an empirical antibiotic treatment, as observed in $11 \%$ of cases included in the study published by Von der Brelie. ${ }^{12}$

A study conducted by Fulkerson, et al. ${ }^{10}$ reported significant differences in the CSF WBC count depending on the type of bacteria; however, 
it is worth noting the high cellularity observed for both Gram-positive and Gram-negative microorganisms. As seen in our study, there is no description in relation to the time elapsed from symptom onset to infection diagnosis (given the difficulty to collect such information in retrospective studies); if a long time has elapsed, it may account as one of the causes for such significant increase in CSF cellularity.

Some series have also reported increased CSF eosinophil count in children with pyogenic ventriculitis, although such finding is rare. CSF eosinophil counts are associated to leak and presence of blood in the CSF, and allergies to silicone and other materials, in addition to infections. ${ }^{13,14}$ Eosinophilia is described as the presence of at least one eosinophil per field, since none should be present. In our review, no patient had eosinophilia.

Another outcome measure previously studied in order to predict the type of bacteria that may be causing pyogenic ventriculitisis the time elapsed between shunt placement and infection onset; however, its usefulness has not been demonstrated in our research. The article published by McGirt ${ }^{15}$ is consistent with our results: bacteria identified in the first 14 days following VPS placement were the same as those observed after this period.

One of the limitations of our research, also observed in published studies, is the small number of patients with this infection, resulting in a small sample size that may influence the statistical power to find group differences. Another limitation of our work is its retrospective nature, which hinders the analysis of certain outcome measures, such as patient symptoms.

TABLE 1. Characteristics of cerebrospinal fluid in pyogenic ventriculitis based on Gram staining $(n=32)$

\begin{tabular}{lccc}
\hline & $\begin{array}{c}\text { Gram } \\
\text { positive }^{1}\end{array}$ & $\begin{array}{c}\text { Gram } \\
\text { negative }^{1}\end{array}$ & P $^{*}$ \\
\hline CSF glucose level mg/dL & 49.5 & 16 & 0.14 \\
& $(0-96)$ & $(0-84)$ & \\
CSF protein level mg/dL & 54.5 & 117.5 & 0.17 \\
& $(10-684)$ & $(10-320)$ & \\
$\mathrm{WBC} / \mathrm{mm}^{3}$ & 21 & 70 & 0.79 \\
& $(0-1900)$ & $(2-240)$ & \\
\hline
\end{tabular}

* Mann-Whitney.

${ }^{1}$ Median (range).

\section{CONCLUSION}

Gram-positive bacteria were widely predominant as a cause of pyogenic ventriculitis associated to the surgical procedure. Unlike previous studies, CSF findings show a high dispersion and were not associated to the type of microorganism in this studied sample. At present, CSF cytochemistry may not help to guide microbiological diagnosis or be useful to decide on an initial empirical antibiotic treatment.

\section{REFERENCES}

1. Sakka L, Coll G, Chazal J. Anatomy and physiology of cerebrospinal fluid. Eur Ann Otorhinolaryngol Head Neck Dis 2011;128(6):309-16.

2. Stenehjem E,Armstrong WS. Centralnervous system device infections. Infect Dis Clin North Am 2012;26(1):89-110.

3. Grandi L, Goldsman A, Jaimovich J, Cohen Arazi J. Pioventriculitis asociada a derivaciones ventriculares. Med Infant 1997;4(1):7-10.

4. Lee P, DiPatri AJ. Evaluation of suspected cerebrospinal fluid shunt complications in children. Clin Pediatr Emerg Med 2008;9(2):76-82.

5. Pfisterer W, Mühlbauer M, Czech T, Reinprecht A. Early diagnosis of external ventricular drainage infection: results of a prospective study. J Neurol Neurosurg Psychiatry 2003;74(7):929-32.

6. Centers for Disease Control and Prevention. CDC/ NHSN Surveillance definitions for specific types of infections. Atlanta: CDC; 2014. [Accessed on: December 4, 2014]. Available at: http:/ / www.cdc.gov/nhsn/PDFs / pscManual/17pscNosInfDef_current.pdf.

7. Wang KW, Chang WN, Shih TY, Huang CR, et al. Infection of cerebrospinal fluid shunts: causative pathogens, clinical features, and outcomes. Jpn J Infect Dis 2004;57(2):44-8.

8. McClinton D, Carraccio C, Englander R. Predictors of ventriculoperitoneal shunt pathology. Pediatr Infect Dis J 2001;20(6):593-7.

9. Muttaiyah S, Ritchie S, Upton A, Roberts S. Clinical parameters do not predict infection in patients with external ventricular drains: a retrospective observational study of daily cerebrospinal fluid analysis. J Med Microbiol 2008;57(Pt 2):207-9.

10. Fulkerson DH, Sivaganesan A, Hill JD, Edwards JR, et al. Progression of cerebrospinal fluid cell count and differential over a treatment course of shunt infection. I Neurosurg Pediatr 2011;8(6):613-9.

s Rosanova MT, Paganini H. Infecciones asociadas a sistemas de derivación de líquido cefalorraquídeo. Med Infant 1997;4(1):26-30.

12. Von der Brelie C, Simon A, Gröner A, Molitor E, et al. Evaluation of an institutional guideline for the treatment of cerebrospinal fluid shunt-associated infections. Acta Neurochir (Wien) 2012;154(9):1691-7.

13. Bezerra S, Frigeri TM, Severo CM, Santana JC, et al. Cerebrospinal fluid eosinophilia associated with intraventricular shunts. Clin Neurol Neurosurg 2011;113(5):345-9.

14. Fulkerson DH, Boaz JC. Cerebrospinal fluid eosinophilia in children with ventricular shunts. J Neurosurg Pediatr 2008;1(4):288-95.

15. McGirt MJ, Zaas A, Fuchs HE, George TM, et al. Risk factors for pediatric ventriculoperitoneal shunt infection and predictors of infectious pathogens. Clin Infect Dis 2003;36(7):858-62. 